\title{
Frailty increases mortality among patients $\geq 80$ years old treated in Polish ICUs
}

Jakub Fronczek ${ }^{1}$, Kamil Polok ${ }^{1}$, Ilona Nowak-Kózka ${ }^{1}$, Anna Włudarczyk¹, Jacek Górka ${ }^{1}$, Mirosław Czuczwar², Paweł Krawczyk ${ }^{3}$, Mirosław Ziętkiewicz ${ }^{3,4}$, Łukasz R. Nowak' Maciej Żukowski ${ }^{6}$, Katarzyna Kotfis $^{6}$, Katarzyna Cwyl ${ }^{7}$, Ryszard Gajdosz ${ }^{8,9}$, Romuald Bohatyrewicz ${ }^{10}$, Jowita Biernawska ${ }^{10}$, Paweł Grudzień ${ }^{11}$, Paweł Nasiłowski ${ }^{12}$, Natalia Popek ${ }^{13}$, Waldemar Cyrankiewicz ${ }^{14}$, Katarzyna Wawrzyniak ${ }^{14}$, Marek Wnuk ${ }^{15}$, Dariusz Maciejewski ${ }^{16}$, Dorota Studzińska ${ }^{17}$, Szymon Bernas ${ }^{18}$, Mariusz Piechota ${ }^{18}$, Waldemar Machała ${ }^{19}$, Marta Serwa ${ }^{19}$, Maria Wujtewicz ${ }^{20}$, Jan Stefaniak ${ }^{20}$, Małgorzata Szymkowiak ${ }^{21}$, Ryszard Gawda ${ }^{22}$, Barbara Adamik ${ }^{23}$, Natalia Kozera ${ }^{23}$, Waldemar Goździk ${ }^{23}$, Hans Flaatten ${ }^{24}$, Wojciech Szczeklik ${ }^{1}$

${ }^{1}$ Department of Intensive Care and Perioperative Medicine, Jagiellonian University Medical College, Cracow, Poland

${ }^{2} 2^{\text {nd }}$ Department of Anesthesiology and Intensive Care Medical University of Lublin, Poland

${ }^{3}$ Department of Anaesthesiology and Intensive Care Medicine, Jagiellonian University Medical College, Cracow, Poland

${ }^{4}$ Thoracic Anaesthesia and Respiratory ICU, John Paul II Hospital, Kraków, Poland

${ }^{5}$ Maria Skłodowska-Curie Memorial Institute of Oncology, Cancer Centre, Krakow, Poland

${ }^{6}$ Department of Anesthesiology, Intensive Therapy and Acute Intoxications, University Hospital No. 2,

Pomeranian Medical University in Szczecin, Poland

${ }^{7}$ Anesthesia and Intensive Care Unit, Regional Health Center in Lubin, Poland

${ }^{8}$ Faculty of Medicine and Health Sciences Krakow University A.F. Modrzewski, Poland

${ }^{9}$ St. Raphael Hospital, Department of Anaesthesiology and Intensive Care, Cracow, Poland

${ }_{10}$ Pomeranian Medical University SPSK 1, Clinical Anesthesiology and Intensive Therapy PUM, Poland

${ }^{11}$ Department of Anaesthesiology and Intensive Therapy, E. Szczeklik Specialist Hospital in Tarnów, Poland

${ }^{12}$ Department of Anaesthesiology and Intensive Therapy, G. Narutowicz Specialist Hospital in Cracow, Poland

${ }^{13}$ Department of Anaesthesiology and Intensive Therapy, S. Żeromski Specialist Hospital in Cracow, Poland

${ }^{14}$ Department of Anaesthesiology and Intensive Therapy, Antoni Jurasz University Hospital No. 1 in Bydgoszcz, Poland

${ }^{15}$ Department of Aanesthesiology and Intensive Therapy, John Paul II Regional Hospital in Bełchatów, Poland

${ }^{16}$ Faculty of Health Science, ATH Bielsko-Biała and Department of Ansesthesiology and Intensive Therapy,

Regional Hospital in Bielsko-Biała, Poland

${ }^{17}$ Department of Anesthesiology and Intensive Care, St John Grande Hospital in Cracow, Poland

${ }^{18}$ Department of Anaesthesiology and Intensive Therapy - Centre for Artificial Extracorporeal Kidney

and Liver Support, The Dr Wt. Biegański Regional Specialist Hospital in Lodz, Poland

${ }^{19}$ Anaesthesia and Intensive Care Clinic, Central Clinical Hospital CKD, University Medical College in Lodz, Poland

${ }^{20}$ Department of Anaesthesiology and Intensive Therapy, Faculty of Medicine, Medical University of Gdańsk, Poland

${ }^{21}$ Department of Anaesthesiology and Intensive Therapy, J. Struś Specialist Hospital in Poznań, Poland

${ }^{22}$ Department of Anesthesiology and Intensive Care, Opole University Hospital, Poland

${ }^{23}$ Department of Anesthesiology and Intensive Therapy, Wroclaw Medical University, Poland

${ }^{24}$ Department of Anesthesia and Intensive Care, Haukeland University Hospital, Norway 


\begin{abstract}
Background: The increasing population of very old intensive care patients (VIPs) is a major challenge currently faced by clinicians and policymakers. Reliable indicators of VIPs' prognosis and appropriateness of their admission to the intensive care unit (ICU) are urgently needed.
\end{abstract}

Methods: This is a report from the Polish sample of the VIP1 multicentre cohort study (NCT03134807). Patients $\geq 80$ years of age admitted to the ICU were included in the study. Information on the type and reason for admission, demographics, utilisation of ICU procedures, ICU length of stay, organ dysfunction and the decision to apply end-of-life care was collected. The primary objective was to investigate the impact of frailty syndrome on ICU and 30-day survival of VIPs. Frailty was assessed with the Clinical Frailty Scale ( $\geq 5$ points on a scale of 1-9).

Results: We enrolled 272 participants with a median age of 84 (81-87) years. Frailty was diagnosed in 170 (62.5\%) patients. The ICU and 30 -day survival rates were equal to $54.6 \%$ and $47.3 \%$ respectively. Three variables were found to significantly increase the odds of death in the ICU in a multiple logistic regression model, namely: SOFA score (OR = 1.16; $95 \% \mathrm{Cl}: 1.16-1.24)$; acute mode of admission ( $\mathrm{OR}=5.1 ; 95 \% \mathrm{Cl}: 1.67-15.57)$; and frailty $(\mathrm{OR}=2.25 ; 95 \% \mathrm{Cl}: 1.26-4.01)$. Conclusion: Measuring frailty in critically ill older adults can facilitate making more informed clinical decisions and help avoid futile interventions.

Anaesthesiology Intensive Therapy 2018, vol. 50, no 4, 245-251

Key words: frailty, intensive care units, medical futility, critical care

It is estimated that by the year 2050 people at the age of 80 years and older will represent almost $10 \%$ of the European population [1]. Progressive ageing remains one of the leading issues in contemporary critical care [2]. The increasing proportion of elderly patients admitted to ICUs requires substantial resources, while many countries are facing a shortage of ICU beds [3-5]. Although our knowledge concerning outcomes in this population is growing, there are currently no local reports from Poland $[6,7]$.

The long-term prognosis of "very old intensive care patients" (VIPs) is often poor, which requires careful weighing of potential costs and benefits associated with intensive care [8]. There is a growing recognition of the fact that clinical features used in traditional disease severity scores, such as APACHE and SAPS, may be insufficient to accurately predict the outcome in VIPs [2]. Population-specific conditions, such as sarcopenia, dementia, delirium and frailty, could be incorporated into the evaluation of elderly patients in the setting of critical illness in order to help better define their prospects of recovery [9-12].

Of the above-mentioned factors, frailty syndrome seems to be the most reliable indicator of biological age [13]. Frailty is defined as a clinically recognisable state of increased vulnerability resulting from ageing-associated decline in reserve and function across multiple physiologic systems such that the ability to cope with everyday or acute stressors is compromised [14]. Its impact on patients' outcomes is established in geriatric and surgical settings, with less evidence available in the field of intensive care. A systematic review and meta-analysis by Muscedere et al. [15] showed a significant impact of frailty on hospital and long-term mor- tality in critically ill patients. However, the relevance of these results is limited by a moderate quality of included studies.

The Very Old Intensive Care Patient: A Multinational Prospective Observation Study (VIP1) was designed to reliably address this issue. Its main goal was to assess the prevalence of frailty among VIPs and assess its impact on their outcomes in the ICU. In the current paper, we would like to present results of the VIP1 study based on Polish population with a particular interest in frailty and life-sustaining treatment limitations, further referred to as end-of-life care interchangeably.

\section{METHODS}

The study was approved by the local ethics committee (Jagiellonian University Ethics Committee), while the protocol complied with the Declaration of Helsinki. According to ethics committee's verdict, patient consent was not required.

VIP1 was a prospective multicentre study coordinated by the Health Services Resource and Outcome (HSRO) section of the European Society of Intensive Care Medicine (ESICM). The enrolment and data collection processes were described in detail in the original VIP1 Study [16]. In this paper, we reported data from the Polish VIP1 cohort.

Patients of 80 years of age or older admitted to Polish ICUs were considered eligible for the study. We collected information on the mode and reason for admission, demographics, utilisation of ICU procedures (i.e. type of respiratory support, administration of vasoactive drugs, initiation of renal replacement therapy), ICU length of stay, organ dysfunction assessed with SOFA score and the decision to 


\section{Clinical Frailty Scale*}

1 Very Fit - People who are robust, active, energetic and motivated. These people commonly exercise regularly. They are among the fittest for their age.

2 Well - People who have no active disease symptoms but are less fit than category I. Often, they exercise or are very active occasionally, e.g. seasonally.

3 Managing Well - People whose medical problems are well controlled, but are not regularly active beyond routine walking.

4 Vulnerable - While not dependent on others for daily help, often symptoms limit activities. A common complaint is being "slowed up", and/or being tired during the day.

5 Mildly Frail - These people often have more evident slowing, and need help in high order IADLs (finances, transportation, heavy housework, medications). Typically, mild frailty progressively impairs shopping and walking outside alone, meal preparation and housework.

6 Moderately Frail - People need help with all outside activities and with keeping house. Inside, they often have problems with stairs and need help with bathing and might need minimal assistance (cuing, standby) with dressing.
榲

7 Severely Frail - Completely dependent for personal care, from whatever cause (physical or cognitive). Even so, they seem stable and not at high risk of dying (within $\sim 6$ months).

8 Very Severely Frail - Completely dependent, approaching the end of life. Typically, they could not recover even from a minor illness.

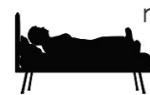

9.Terminally III - Approaching the end of life.This category applies to people with a life expectancy $<6$ months, who are not otherwise evidently frail.

\section{Scoring frailty in people with dementia}

The degree of frailty corresponds to the degree of dementia. Common symptoms in mild dementia include forgetting the details of a recent event, though still remembering the event itself, repeating the same question/story and social withdrawal.

In moderate dementia, recent memory is very impaired, even though they seemingly can remember their past life events well. They can do personal care with prompting

In severe dementia, they cannot do personal care without help.

* I. Canadian Study on Health \& Aging, Revised 2008

2. K. Rockwood et al.A global clinical measure of fitness and frailty in elderly people. CMAJ 2005; 173:489-495.

(c) 2007-2009.Version 1.2.All rights reserved. Geriatric Medicine Research, Dalhousie University, Halifax, Canada. Permission granted to copy for research and educational purposes only.

Figure 1. Clinical Frailty Scale

introduce end-of-life care (withholding and/or withdrawal of further treatment). Specific definitions had been previously described elsewhere [16].

The main objective of the study was to investigate the ICU and 30-day survival in relation to the presence of frailty syndrome before the onset of acute illness and to characterise the intensity of treatment in this group. Frailty was assessed with the Clinical Frailty Scale (CFS), a simple tool used by the ICU staff according to a visual description to categorise patients as frail or non-frail ( $\geq 5$ points or $<5$ points respectively on a scale of $1-9$ ) (Fig. 1) [17].

Continuous variables were described as medians with interquartile ranges, categorical variables were reported as percentage values. The Mann-Whitney $U$ test and chisquared test (Pearson's chi-squared or Fisher's exact test) were used in the between-groups univariate analyses as applicable. The effect size was represented by odds ratios with $95 \%$ confidence intervals. A multiple logistic regression model was used to report adjusted odds ratios for the ICU mortality of different clinical characteristics. $P$-values $<0.05$ were considered statistically significant.

\section{RESULTS}

Between November 2016 and February 2017, 272 participants were enrolled in the study. The overall ICU and 30-day survival rates were equal to $54.6 \%$ and $47.3 \%$ respectively. Data on ICU survival was available in 269 (98.9\%) patients, while 239 (87.9\%) individuals completed the 30-day follow-up. Frailty was diagnosed in 170 (62.5\%) patients admitted to ICUs.

The median age of the studied population was 84 (8187) years and $159(58.5 \%)$ patients were female. The median SOFA score on admission was equal to 10 (7-14). The CFS score used to assess frailty reached a median value of 5 (4-6.75), while the median length of stay in the ICU was 4 days (1.3-15.9).

Most admissions were classified as acute, with only 42 (15.4\%) patients transferred to the ICU after elective surgery. There were $48(17.6 \%)$ patients who stayed less than $24 \mathrm{~h}$ in the ICU (one-day stay). The median time spent in hospital before admission to the ICU was 2 days (0-5). The most common baseline diagnosis was a combination of circulatory and respiratory failure, reported in 72 (26.5\%) cases. The majority of patients required intubation, mechanical ventilation and administration of vasoactive drugs (Table 1). 
Table 1. Differences in clinical characteristics between frail and non-frail patients

\begin{tabular}{|c|c|c|c|}
\hline Characteristic & $\begin{array}{c}\text { Not frail } \\
n=102\end{array}$ & $\begin{array}{c}\text { Frail } \\
n=170\end{array}$ & P-value \\
\hline Age (years) & $83(81-85.25)$ & $85(82-88)$ & 0.004 \\
\hline SOFA score & $10(6-14)$ & $10(7-14)$ & 0.99 \\
\hline Gender (female) & $57.8 \%$ & $58.8 \%$ & 0.87 \\
\hline Non-invasive ventilation & $7.8 \%$ & $15.9 \%$ & 0.06 \\
\hline Intubation \& mechanical ventilation & $85.3 \%$ & $84.7 \%$ & 0.90 \\
\hline Vasoactive drugs & $65.7 \%$ & $74.7 \%$ & 0.11 \\
\hline Renal replacement therapy & $21.6 \%$ & $19.4 \%$ & 0.67 \\
\hline Respiratory failure & $19.6 \%$ & $18.2 \%$ & 0.78 \\
\hline Combined circulatory \& respiratory failure & $23.5 \%$ & $28.2 \%$ & 0.39 \\
\hline Post-elective surgery & $20.6 \%$ & $12.4 \%$ & 0.07 \\
\hline One-day stay $(<24$ h at ICU) & $19.6 \%$ & $16.5 \%$ & 0.51 \\
\hline End-of-life care* & $9.8 \%$ & $16.5 \%$ & 0.13 \\
\hline Withholding of treatment & $6.9 \%$ & $8.8 \%$ & 0.57 \\
\hline Withdrawal of treatment & $2.9 \%$ & $7.6 \%$ & 0.18 \\
\hline ICU length of stay (days) & $5.7(1.1-17.3)$ & $3.1(1.3-13.1)$ & 0.26 \\
\hline ICU survival & $66.3 \%$ & $47.6 \%$ & 0.003 \\
\hline 30-day survival ${ }^{\S}$ & $59.1 \%$ & $40.4 \%$ & 0.005 \\
\hline
\end{tabular}

SOFA: Sequential Organ Failure Assessment; ICU: Intensive Care Unit; *End-of-life care: the overall proportion of patients with only withholding and patients with and

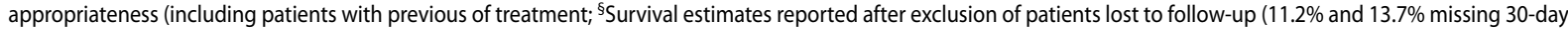
observations of frail and non-frail patients respectively)

Table 2. Multiple logistic regression model - mortality in the ICU

\begin{tabular}{|c|c|c|c|c|}
\hline Characteristic & Estimate & SE & P-value & Adjusted OR $(95 \% \mathrm{Cl})$ \\
\hline SOFA score & 0.15 & 0.03 & $<0.001$ & $1.16(1.09-1.24)$ \\
\hline Frailty & 0.81 & 0.30 & 0.006 & $2.25(1.26-4.01)$ \\
\hline Acute admission & 1.63 & 0.57 & 0.004 & $5.10(1.67-15.57)$ \\
\hline Age (years) & 0.042 & 0.037 & 0.25 & $1.04(0.97-1.12)$ \\
\hline Gender (female) & 0.15 & 0.28 & 0.58 & $1.17(0.67-2.02)$ \\
\hline Intercept & -8.96 & 3.19 & 0.005 & - \\
\hline
\end{tabular}

SOFA: Sequential Organ Failure Assessment

In univariate analyses, patients with frailty syndrome were older and had a significantly lower ICU and 30-day survival rate than those without frailty (Table 1). The unadjusted OR for death in the ICU associated with frailty was equal to 2.17 (95\% Cl: 1.3-3.62; $P=0.003$ ), with a similar effect on 30-day mortality and an OR of $2.13(95 \% \mathrm{Cl}$ : 1.26-3.64, $P=$ 0.005). There were no differences in terms of the baseline SOFA score and utilisation of ICU procedures based on the presence of frailty. End-of-life care was applied in $16.5 \%$ and $9.8 \%$ of the frail and non-frail patients respectively $(P=0.13)$.

Three variables that significantly increased the odds of ICU death were identified in the multiple logistic regression model, namely: SOFA score (OR $=1.16 ; 95 \% \mathrm{Cl}$ : 1.16-1.24); acute mode of admission ( $\mathrm{OR}=5.1 ; 95 \% \mathrm{Cl}$ : 1.67-15.57); and frailty (OR $=2.25 ; 95 \% \mathrm{Cl}: 1.26-4.01$ ) (Table 2). Age was not a significant predictor of poor outcome after adjustment for other clinical characteristics. An analogous model for 30-day mortality exhibited similar findings (results not shown).

An exploratory model investigating whether patients lost to follow-up were more severely ill was provided to verify the possibility of attrition bias. We found that a combination of SOFA score, frailty and type of admission could not predict which patients would leave the study (none of these features achieved statistical significance; $A U C=0.56$ ). 


\section{DISCUSSION}

We reported data of 272 very old critically ill patients admitted to 27ICUs that took part in the VIP1 Study in Poland [16]. The main objective of this multicentre, international study was to investigate prospectively the impact of frailty on short-term mortality and to describe the level of care in this population.

Frailty, assessed with a simple screening tool, was associated with an over two-fold increase in the odds of death independantly of the patient's age and baseline severity of organ failure. The utilisation of medical procedures was similar in patients with and without frailty, and there was no statistically significant difference between both groups in terms of life-sustaining treatment limitations.

The study had a limited sample size due to reporting partial data from a larger cohort study. This did not guarantee sufficient power to detect statistically significant differences in some characteristics and reduced the cohort's representativeness of the population - Polish VIPs had more severe organ dysfunction based on the SOFA score as well as a lower ICU and 30-day survival rate than the average reported in the VIP1 Study. These observations confirm previous reports showing that patients admitted to Polish ICUs are in poor clinical condition [18]. However, the observed mortality has recently been demonstrated to be lower than predicted by the APACHE II model, which might indicate a relatively good performance of ICUs in Poland [19].

In the ageing population, the number of critically ill elderly patients is increasing [20]. Patients $\geq 80$ years old account for $10 \%$ to $20 \%$ of all admissions to the ICU, posing a global challenge to healthcare systems [21]. Limited resources necessitate careful consideration of the potential reversibility of critical illness to avoid futile interventions $[11,22,23]$. In a recent study in JAMA, Guidet et al. [7] have shown that a proactive strategy of admitting elderly patients to the ICU (i.e. an ICU triage) doubled the number of admissions to French ICUs and, at the same time, increased hospital mortality. These findings give rise to a question whether we could better predict which VIPs will, in fact, benefit from intensive care. Traditional disease severity scores do not capture important data on a patient's premorbid condition, such as cognitive impairment, decreased functional capacity and frailty - all described as potential modifiers of morbidity and mortality in VIPs [2].

The concept of assessing patients' biological rather than chronological age is appealing and paves its way from geriatrics to intensive care. Our results support including frailty in the assessment of critically ill elderly patients to improve the discrimination between individuals fit for additional interventions and those in whom withholding or withdrawal of treatment should be considered. This, in turn, should help physicians, along with patients and their families, make more informed decisions concerning the intensity of treatment, its appropriateness and further course. A study which was recently published by Guidet [24] placed frailty among the most influential factors with regard to life-sustaining treatment limitations, along with acute admission, age and SOFA score. The results of the upcoming VIP2 study will help establish a predictive model designed specifically for the critically ill elderly population, which will take into account the above-mentioned features [25]. Despite being calculated with a dedicated tool, risk estimates alone are unlikely to guide treatment in the ICU. However, accurate outcome prediction could facilitate clinical judgement and improve the communication between providers and recipients of care.

A decision to de-escalate treatment in a particular case is never an easy one. In the Polish cohort of VIPs, a decision to introduce end-of-life care was made in $14 \%$ of the patients, compared to $27.2 \%$ reported across all centres participating in the study. These numbers go along with results of the paper published by Guidet [24], which showed a lower frequency of life-sustaining treatment limitation in Eastern Europe. While it does not allow for making direct inferences, we should be wary about the fact that many patients approaching the end of life tend to value comfort over aggressive treatment when given the opportunity to express their preferences [26]. The evidence suggests that this an often underappreciated practice in the ICU, where the patient's opinion is rarely sought, and the preferences of the family are discordant with the care provided in many cases [27-29]. Despite the potential short-term benefit of treatment in the ICU, the long-term outcome in this population is unfavourable [30-32].

Maintenance of life-support measures in patients who have no real prospects of recovery is a broadly discussed topic. Polish guidelines on intensive care referring to this issue considered futile medical therapy as malpractice and have highlighted the role of palliative care in this setting [33]. This is an important step towards overcoming the legal, ethical and religious reservations which may all affect the decision whether to de-escalate or discontinue further treatment [24, 34]. The above-mentioned factors are likely to be more pronounced in Polish intensivists compared to nationalities with a less conservative attitude [35]. In many cases, the death of a critically ill elderly patient is a natural history of disease rather than a therapeutic failure. Amid the flurry of intensive care, we can all benefit from acknowledging end-of-life care $[36,37]$.

Nevertheless, we do not all age the same. An octogenarian may still have his greatest mountains to climb ahead - just like the famous Mr. Miura, who at the age of eighty, against all odds, reached the summit Mount Everest for the third time in his life [38]. Thus, adopting the assessment of 
frailty in critical care has a potential to identify patients who are more likely to recover from an acute illness and to act upon it with an adequate intensity of intensive care.

\section{CONCLUSIONS}

The presence of frailty increases mortality among critically ill patients $\geq 80$ years old treated in Polish ICUs. Measuring frailty in this population can facilitate making more informed clinical decisions and help avoid futile interventions.

\section{ACKNOWLEDGEMENTS}

1. Financial support: the study was sponsored by University of Bergen.

2. Conflict of interest: none.

\section{References:}

1. Nguyen $\mathrm{YL}$, Angus DC, Boumendil A, et al. The challenge of admitting the very elderly to intensive care. Ann Intensive Care. 2011; 1(1):29, doi: 10.1186/2110-5820-1-29, indexed in Pubmed: 21906383.

2. Flaatten $\mathrm{H}$, de Lange $\mathrm{DW}$, Artigas $\mathrm{A}$, et al. The status of intensive care medicine research and a future agenda for very old patients in the ICU. Intensive Care Med. 2017; 43(9): 1319-1328, doi: 10.1007/s00134-0174718-z, indexed in Pubmed: 28238055.

3. Stelfox HT, Hemmelgarn BR, Bagshaw SM, et al. Intensive care unit bed availability and outcomes for hospitalized patients with sudden clinical deterioration. Arch Intern Med. 2012; 172(6): 467-474, doi: 10.1001/ archinternmed.2011.2315, indexed in Pubmed: 22412076.

4. Simchen E, Sprung CL, Galai N, et al. Survival of critically ill patients hospitalized in and out of intensive care units under paucity of intensive care unit beds. Crit Care Med. 2004; 32(8): 1654-1661, indexed in Pubmed: 15286540

5. Town JA, Churpek MM, Yuen TC, et al. Relationship between ICU bed availability, ICU readmission, and cardiac arrest in the general wards. Crit Care Med. 2014; 42(9): 2037-2041, doi: 10.1097/ CCM.0000000000000401, indexed in Pubmed: 24776607.

6. Bagshaw SM, Webb SAR, Delaney A, et al. Very old patients admitted to intensive care in Australia and New Zealand: a multi-centre cohort analysis. Crit Care. 2009; 13(2): R45, doi: 10.1186/cc7768, indexed in Pubmed: 19335921.

7. Guidet B, Leblanc G, Simon T, et al. ICE-CUB 2 Study Network. Effect of systematic intensive care unit triage on long-term mortality among critically III elderly patients in france: a randomized clinical trial. JAMA. 2017; 318(15): 1450-1459, doi: 10.1001/jama.2017.13889, indexed in Pubmed: 28973065.

8. Guidet B, de Lange DW, Flaatten $\mathrm{H}$. Should this elderly patient be admitted to the ICU? Intensive Care Med. 2018 [Epub ahead of print], doi: 10.1007/s00134-018-5054-7, indexed in Pubmed: 29356853.

9. Bagshaw SM, Stelfox HT, Johnson JA, et al. Long-term association between frailty and health-related quality of life among survivors of critical illness: a prospective multicenter cohort study. Crit Care Med. 2015; 43(5): 973-982, doi: 10.1097/CCM.0000000000000860, indexed in Pubmed: 25668751.

10. Le Maguet $P$, Roquilly $A$, Lasocki $S$, et al. Prevalence and impact of frailty on mortality in elderly ICU patients: a prospective, multicenter, observational study. Intensive Care Med. 2014; 40(5): 674-682, doi: 10.1007/s00134-014-3253-4, indexed in Pubmed: 24651884.

11. Boumendil A, Angus DC, Guitonneau AL, et al. ICE-CUB study group. Variability of intensive care admission decisions for the very elderly. PLoS One. 2012; 7(4): e34387, doi: 10.1371/journal.pone.0034387, indexed in Pubmed: 22509296.

12. Poole D, Finazzi S, Nattino G, et al. GiViTI Group. The prognostic importance of chronic end-stage diseases in geriatric patients admitted to 163 Italian ICUs. Minerva Anestesiol. 2017; 83(12): 1283-1293, doi: 10.23736/S0375-9393.17.11919-X, indexed in Pubmed: 28631455.

13. Vidal EIO, Mayoral VFS, Villas Boas PJF, et al. Physical frailty as a clinical marker of biological age and aging. J Am Geriatr Soc. 2015; 63(4): 837-838, doi: 10.1111/jgs.13356, indexed in Pubmed: 25900511.
14. Xue QL. The frailty syndrome: definition and natural history. Clin Geriatr Med. 2011; 27(1): 1-15, doi: 10.1016/j.cger.2010.08.009, indexed in Pubmed: 21093718.

15. Muscedere J, Waters B, Varambally A, et al. The impact of frailty on intensive care unit outcomes: a systematic review and meta-analysis. Intensive Care Med. 2017; 43(8): 1105-1122, doi: 10.1007/s00134-0174867-0, indexed in Pubmed: 28676896.

16. Flaatten $\mathrm{H}$, De Lange DW, Morandi A, et al. VIP1 study group. The impact of frailty on ICU and 30-day mortality and the level of care in very elderly patients ( $\geq 80$ years). Intensive Care Med. 2017; 43(12): 1820-1828, doi: 10.1007/s00134-017-4940-8, indexed in Pubmed: 28936626.

17. Rockwood K, Song X, MacKnight C, et al. A global clinical measure of fitness and frailty in elderly people. CMAJ. 2005; 173(5): 489-495, doi: 10.1503/cmaj.050051, indexed in Pubmed: 16129869.

18. Vincent JL, Marshall JC, Namendys-Silva SA, et al. ICON investigators. Assessment of the worldwide burden of critical illness: the intensive care over nations (ICON) audit. Lancet Respir Med. 2014; 2(5): 380-386, doi: 10.1016/S2213-2600(14)70061-X, indexed in Pubmed: 24740011.

19. Knapik P, Krzych $\succeq J$, Weigl W, et al. Mortality rate in Polish intensive care units is lower than predicted according to the APACHE II scoring system. Intensive Care Med. 2017; 43(11): 1745-1746, doi: 10.1007/ s00134-017-4883-0, indexed in Pubmed: 28733717.

20. Ihra GC, Lehberger J, Hochrieser $\mathrm{H}$, et al. Development of demographics and outcome of very old critically ill patients admitted to intensive care units. Intensive Care Med. 2012; 38(4): 620-626, doi: 10.1007/s00134012-2474-7, indexed in Pubmed: 22354500.

21. Chin-Yee N, D'Egidio G, Thavorn K, et al. Cost analysis of the very elderly admitted to intensive care units. Crit Care. 2017; 21(1): 109, doi: 10.1186/ s13054-017-1689-y, indexed in Pubmed: 28506243.

22. Heyland D, Cook D, Bagshaw SM, et al. Canadian Critical Care Trials Group, Canadian Researchers at the End of Life Network. The Very Elderly Admitted to ICU: A Quality Finish? Crit Care Med. 2015; 43(7): 1352-1360, doi: 10.1097/CCM.0000000000001024, indexed in Pubmed: 25901550.

23. Sprung $\mathrm{CL}$, Artigas A, Kesecioglu J, et al. The Eldicus prospective, observational study of triage decision making in European intensive care units. Part II: intensive care benefit for the elderly. Crit Care Med. 2012; 40(1): 132-138, doi: 10.1097/CCM.0b013e318232d6b0, indexed in Pubmed: 22001580.

24. Guidet B, Flaatten $\mathrm{H}$, Boumendil A, et al. VIP1 study group. Withholding or withdrawing of life-sustaining therapy in older adults ( $\geq 80$ years) admitted to the intensive care unit. Intensive Care Med. 2018; 44(7): 1027-1038, doi: 10.1007/s00134-018-5196-7, indexed in Pubmed: 29774388.

25. https://www.vip2study.com. (30.06.2018).

26. Philippart F, Vesin A, Bruel C, et al. The ETHICA study (part I): elderly's thoughts about intensive care unit admission for life-sustaining treatments. Intensive Care Med. 2013; 39(9): 1565-1573, doi: 10.1007/ s00134-013-2976-y, indexed in Pubmed: 23765236.

27. Le Guen J, Boumendil A, Guidet B, et al. Are elderly patients' opinions sought before admission to an intensive care unit? Results of the ICE-CUB study. Age Ageing. 2016; 45(2): 303-309, doi: 10.1093/ageing/ afv191, indexed in Pubmed: 26758531.

28. Heyland DK, Dodek P, Mehta S, et al. Canadian Critical Care Trials Group and Canadian Researchers at End of Life Network (CARENET). Admission of the very elderly to the intensive care unit: family members' perspectives on clinical decision-making from a multicenter cohort study. Palliat Med. 2015; 29(4): 324-335, doi: 10.1177/0269216314566060, indexed in Pubmed: 25645668.

29. Heyland DK, Barwich D, Pichora D, et al. ACCEPT (Advance Care Planning Evaluation in Elderly Patients) Study Team, Canadian Researchers at the End of Life Network (CARENET). Failure to engage hospitalized elderly patients and their families in advance care planning. JAMA Intern Med. 2013; 173(9): 778-787, doi: 10.1001/jamainternmed.2013.180, indexed in Pubmed: 23545563.

30. Flaatten $\mathrm{H}$, Garrouste-Orgeas M. The very old ICU patient: a never-ending story. Intensive Care Med. 2015; 41(11): 1996-1998, doi: 10.1007/ s00134-015-4052-2, indexed in Pubmed: 26359170.

31. Sprung $\mathrm{CL}$, Artigas A, Kesecioglu J, et al. The Eldicus prospective, observational study of triage decision making in European intensive care units. Part II: intensive care benefit for the elderly. Crit Care Med. 2012; 40(1): 132-138, doi: 10.1097/CCM.0b013e318232d6b0, indexed in Pubmed: 22001580. 
32. Kaarlola A, Tallgren M, Pettilä V. Long-term survival, quality of life, and quality-adjusted life-years among critically ill elderly patients. Crit Care Med. 2006; 34(8): 2120-2126, doi: 10.1097/01. CCM.0000227656.31911.2E, indexed in Pubmed: 16763517.

33. Kübler A, Siewiera J, Durek G, et al. Guidelines regarding the ineffective maintenance of organ functions (futile therapy) in ICU patients incapable of giving informed statements of will. Anaesthesiol Intensive Ther. 2014; 46(4): 215-220, doi: 10.5603/AIT.a2014.0038, indexed in Pubmed: 25293473.

34. Kübler A, Adamik B, Lipinska-Gediga $M$, et al. End-of-life attitudes of intensive care physicians in Poland: results of a national survey. Intensive Care Med. 2011;37(8): 1290-1296, doi: 10.1007/s00134-011-2269-2, indexed in Pubmed: 21660534.

35. Adamski J, Goraj R, Onichimowski D, et al. The differences between two selected intensive care units located in central and northern Europe - preliminary observation. Anaesthesiol Intensive Ther. 2015; 47(2): 117-124, doi: 10.5603/AIT.a2015.0010, indexed in Pubmed: 25772830.

36. Leblanc G, Boumendil A, Guidet B. Ten things to know about critically ill elderly patients. Intensive Care Med. 2017;43(2):217-219, doi: 10.1007/ s00134-016-4477-2, indexed in Pubmed: 27492269.
37. Cardona-Morrell M, Hillman K. Development of a tool for defining and identifying the dying patient in hospital: Criteria for Screening and Triaging to Appropriate aLternative care (CriSTAL). BMJ Support Palliat Care. 2015; 5(1): 78-90, doi: 10.1136/bmjspcare-2014-000770, indexed in Pubmed: 25613983.

38. Szawarski P. Classic cases revisited: Mr Miura and the delusion of immortality. J Intensive Care Soc. 2018; 19(3): 269-273, doi: 10.1177/1751143718754995, indexed in Pubmed: 30159020.

\section{Corresponding author:}

Wojciech Szczeklik, MD, PhD

Jagiellonian University Medical College

Department of Intensive Care and Perioperative Medicine

ul. Skawińska 8, 31-066 Krakow, Poland

e-mail:wojciech.szczeklik@uj.edu.pl

Received: 2.07.2018

Accepted: 21.09.2018 